\title{
FMH, FMH Services und HIN an der IFAS 2016
}

\author{
Christoph Kreyden ${ }^{\text {a }}$, Claudia Blackburn ${ }^{b}$ \\ a Leiter Dienst Dienstleistungen Mitgliedschaft \\ b Verantwortliche Marketing und Kommunikation FMH Consulting Services
}

Die FMH, die FMH Services und HIN heissen Sie gerne an der Internationalen Fachmesse für Arzt- und Spitalbedarf (IFAS) am gemeinsamen Stand auf der Galerie vor der Halle 7 willkommen. Lernen Sie unsere vielfältigen Dienstleistungen in den Bereichen Weiterbildung, sicherer Datenaustausch, Praxisgründung, Praxisführung, Praxisübergabe und Gruppenpraxis kennen! Wir freuen uns auf den persönlichen Kontakt mit Ihnen und gehen gerne auf Ihre Anliegen und Bedürfnisse ein.

\section{Besuchen Sie unsere Workshops am Mittwoch, 26. Oktober 2016, und insbe- sondere am Donnerstag, 27. Oktober 2016}

Am Mittwoch, 26. Oktober 2016, sowie insbesondere am Donnerstag, 27. Oktober 2016, haben Sie Gelegenheit, an unseren Workshops teilzunehmen. Bestimmt finden Sie in unserem Angebot das eine oder andere Thema, das Sie besonders interessiert. Wir freuen uns auf Ihre Anmeldung über www.fmh.ch $\rightarrow$ Services $\rightarrow$ Für die Mitglieder $\rightarrow$ IFAS 2016.

\section{Weitere Informationen}

\section{Veranstaltungsort:}

Die Workshops werden durchgeführt im Seminarraum K6 (Zugang vom Galeriegeschoss vor Halle 7).

\section{Teilnehmerkreis:}

Die FMH- und FMH Services-Workshops stehen ausschliesslich Ärztinnen und Ärzten sowie deren MPAs offen (sofern die Anmeldung durch den Praxisinhaber erfolgt), werden ausschliesslich in deutscher Sprache durchgeführt und sind kostenlos.

Der HIN-Workshop richtet sich an Ärztinnen und Ärzte, IT-Fachpersonen von Institutionen sowie HIN-Partner. Die Registrierung erfolgt nach Eingang der Anmeldungen. Die Teilnahme an den Workshops berechtigt zum Gratiseintritt zur IFAS 2016.

Korrespondenz FMH / Dienst DLM Elfenstrasse 18 Postfach 300 CH-3000 Bern 15 Tel. 0313591111 dlm[at]fmh.ch

\section{Anmeldung:}

Online-Anmeldeformular unter www.fmh.ch $\rightarrow$ Services $\rightarrow$ Für die Mitglieder $\rightarrow$ IFAS 2016.

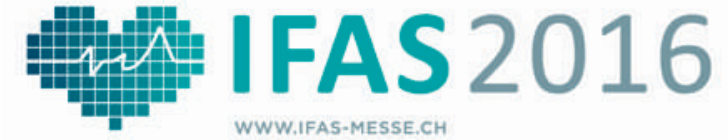

Workshop Nr. 1 (FMH): Aktuelle Fragen rund um die Krankengeschichte

Donnerstag, 27.10.2016, 10:00-11:00 Uhr

Referentinnen: Dr. iur. Caroline Hartmann, Rechtsanwältin, Stv. Leiterin Gutachterstelle FMH / Gabriela Lang, Rechtsanwältin, Stv. Leiterin Abteilung Rechtsdienst FMH

Fokus auf:

- Einsichts- und Kopienrechte des Patienten an der Krankengeschichte

- Aufbewahrungspflicht

- Die Krankengeschichte als Beweismittel in Haftpflichtfällen

Workshop Nr. 2 (FMH Services): Vermögensaufbau für Ärzte welche Strategie soll ich verfolgen? Donnerstag, 27.10.2016, 11:15-12:15 Uhr

Referent: Alexander Bruderer, FMH Insurance Services

Häufig setzen sich Ärzte nur wenig mit der Strategie des Vermögensaufbaus auseinander. Der Arzt führt üblicherweise ein 3.-Säule-Konto und ist bei einer Pensionskasse angeschlossen. Was sind alternative Strategien, welche auch bei Invalidität für ausreichend Kapital sorgen? Wie erhalte ich mir in meiner Vermögensstrategie eine maximale Flexibilität aufrecht? Der Workshop soll zu diesen Themen Ideen liefern.

Workshop Nr. 3 (FMH Services): Stolpersteine bei der Gründung und Führung einer Gruppenpraxis

Donnerstag, 27.10.2016, 13:00-14:00 Uhr

Referent: PatrickTuor, Leiter Beratung, FMH Consulting Services AG Fokus auf:

- Wahl der juristischen Gesellschaftsform

- Rechtliche, steuerliche bzw. mehrwertsteuerliche Aspekte

- Herausforderungen beim Teamaufbau

- Rentabilität und andere finanzielle Aspekte

- Marketing: Auftritt nach innen und aussen

Workshops Nr. 4 \& Nr. 5 (HIN): EPDG - ohne eID kein Zugriff auf das elektronische Patientendossier

Donnerstag, 27.10.2016, 14:15-18:00 Uhr (Workshop Nr. 4) /

Mittwoch, 26.10.2016, 9:00-12:45 Uhr (Workshop Nr. 5)

Referent: Christian Greuter, Geschäftsführer HIN / Stefan Müller

Senior Sales \& Product Manager HIN

Das im Sommer 2015 verabschiedete Bundesgesetz über das elektronische Patientendossier (EPDG) schreibt vor, dass alle Gesundheitsfachpersonen, die auf ein elektronisches Patientendossier zugreifen wollen, eine elektronische Identität (eID) eines zertifizierten Herausgebers für den Zugriff einsetzen müssen. HIN ist bereit und informiert Sie an einem exklusiven Kundenworkshop. Fokus auf:

- HIN eID und Nutzungsmöglichkeiten für Institutionen

Identitäts- und Zugriffsmanagement im EPDG-Kontext

- $\quad$ Aktuelle Case Studies 